\title{
Methylphenidate has Differential Effects on Numerical Abilities in ADHD Children with and without Co-Morbid Mathematical Difficulties
}

\author{
Orly Rubinsten*, Anne-Claude Bedard and Rosemary Tannock \\ Neuroscience and Mental Health Research Program, The Hospital for Sick Children, Toronto, Canada
}

\begin{abstract}
Objective. To investigate effects of methylphenidate (MPH) on numerical performance in children with Attention-Deficit/Hyperactivity Disorder (ADHD) with and without concurrent math difficulties. Method. Data were analyzed from three groups of children with ADHD, who varied in arithmetic abilities. Groups were matched for IQ and reading abilities and classification was based on ICD-10 criteria, using scores on a standardized arithmetic achievement test. Thus, we identified one group with severe difficulties in arithmetic (ADHD+Developmental Dyscalculia; DD), second group with more general and less severe difficulties in arithmetic (ADHD+Mathematical Disabilities; MD), and a third group with good arithmetic abilities (ADHD). All children completed a 10-minute arithmetic task involving subtraction problems, during an acute, randomized, placebo-controlled cross-over trial with three dose levels of methylphenidate (10mg, $15 \mathrm{mg}, 20 \mathrm{mg}$ ). Results. (1) Both ADHD+MD and ADHD+DD were impaired in using strategies that implicate working memory (i.e., borrowing). However, only the ADHD+DD were impaired in using implicit knowledge of quantities (i.e., doing simple subtractions). (2) MPH improved all children's performance of arithmetic procedures (borrowing) that involves working memory, but had no effect on basic numerical skills that involves understanding of quantities. Conclusions. We show clear dissociation of MPH functions: it improves working memory functions but does not improve specific cognitive functions such as quantity manipulation. Moreover, MPH shows decreased efficacy for arithmetic performance in $\mathrm{ADHD}+\mathrm{DD}$, highlighting the need for additional intervention in this subgroup.
\end{abstract}

Key Words: Methylphenidate, developmental dyscalculia, ADHD.

\section{INTRODUCTION}

Attention-deficit/hyperactivity disorder (ADHD, affecting $4 \%$ to $10 \%$ of school age children [1], is associated with substantial academic underachievement in mathematics and reading [2]. For example, recent estimates suggest that $25 \%$ of children with ADHD have comorbid disorder of mathematics [3]. However, in contrast to co-occurring reading difficulties, mathematical difficulties in ADHD have received scant attention from either clinicians or researchers, despite its functional significance. For example, quantitative literacy is a strong predictor of attaining full-time employment [4] that is a major problem among adults with ADHD [5].

Existing studies indicate that children with ADHD exhibit problems in completing arithmetic calculations quickly and accurately [6-8] and that these problems may persist into adulthood [5]. The rates of co-occurrence of mathematical difficulties and ADHD are greater than in the general population [9], but the underlying mechanisms for the overlap are unknown. Some researchers attribute the significant mathematical delays in children with ADHD to attentional, working memory and executive functions impairments needed for calculations [10]. Specifically, in numerical computations every small detail, such as the position of the digit or differences in operational signs is relevant for doing well on tasks. For example, Geary (1993) claimed that forgetting to borrow or borrowing indirectly in a subtraction problem, is mediated

*Address correspondence to this author at the Neurosciences and Mental Health Research Program, Research Institute, The Hospital for Sick Children, 555 University Ave., Toronto, Ontario, Canada; Tel: +011-4168138210; Fax: +011-416-8136564; E-mail: orlyr@bgu.ac.il by poor attentional and active working memory skills. Such general cognitive impairments (i.e., not specific to mathematics) are considered to be integral features of the ADHD syndrome $[11,12]$ and hence, may cause mathematical difficulties in some of these children $[7,13]$. It is also possible, however, that subgroups of children with ADHD may exhibit different underlying mechanisms, including specific deficits in basic numerical processing (e.g., the ability to understand and manipulate quantity), as manifest in children with developmental dyscalculia (DD) [14,15]. Specifically, animals and humans appear to have an innate core number system that is rooted in evolution and permits mental representation of quantities or magnitudes [16,17]. Core numerical abilities are still discernible in older children and adults and form the building blocks for the development of new cognitive skills such as formal and higher mathematical abilities. Dehaene [18], for example, suggested that arithmetic facts like those generated by division or subtraction involve manipulation of quantities (i.e., core system of numbers, e.g., 19). It is reasonable to assume that there are ADHD children whose difficulties arise from deficits in processing quantities as well as executive dysfunctions.

Given the high rates of co-morbidity between mathematical disabilities and ADHD [3] and the widespread use of stimulants as one mainstay treatment for ADHD [20], controlled investigation of the effect of stimulant medications on mathematics is important both clinically and theoretically. Growing evidence indicates that at least some aspects of mathematical performance are responsive to stimulant treatment (e.g., 7, 21,22). However, longer-term effects of stimulant treatment on academic achievement in mathematics re- 
main equivocal [23,24]. Moreover, one recent study reported that stimulant medication had decreased efficacy in treating children with ADHD plus comorbid learning disorders in mathematics [25]. The clinical implications of that study remain unclear, because only one dose level of MPH was used to ascertain treatment efficacy and the clinical consensus determination of response was based on information aggregated across different domains of functioning (behavioral, cognitive, arithmetic performance). Thus, it is unknown whether the decreased efficacy of MPH for this subgroup of children occurred across all outcome measures or was attributable primarily to lack of effect in one domain (e.g., arithmetic performance).

Furthermore, MPH is known to enhance general cognitive functions such as attention [26], spatial attention [27], spatial memory [28], motivation [29], effortful processing [29,30] and effortful semantic processing [31]. All of which are associated with pre-frontal activation. And indeed, it has been shown that MPH may improve weaknesses in catecholamine signaling in the prefrontal cortex of ADHD participants (for review see 32). By contrast, MPH appears to have little or no influence on basic cognitive functions such as phonological processing or simple storage of auditory-verbal information [26]. Moreover, MPH does not seem to modify Parietal activation [32], that is assumed to be involved with quantity processing [33,34].

Hence, the purpose of the study was to investigate the efficacy of MPH on different aspects of arithmetic performance in children with ADHD with or without a concomitant learning disorder in mathematics. Prior to outlining our hypotheses, we first highlight important taxonomies for mathematical difficulties, as well as pivotal advances in the neuroscience and development of numeracy that guided our hypotheses and methodology

\section{Impairments in Mathematics}

The term 'Developmental Dyscalculia'(DD) is often used synonymously with the psychiatric terminology of 'Mathematics Disorder' (DSM IV) and 'Specific Disorder of Arithmetic Skills' (ICD-10), but it differs conceptually from the psychiatric constructs in several ways. First, DD is more closely aligned with the ICD-10 concept of a Specific Disorder in Arithmetic in that it locates the learning problems to the domain of arithmetic and requires reading and spelling skills to be within normal range. Second, most recently DD is understood to reflect a specific disability in basic numerical processing, such as automatic or implicit processing (or understanding) of quantities or numbers [14,35]. Thus, a diagnosis of DD would likely apply to only a subset of individuals with a diagnosis of Mathematics Disorder or Specific Disorder of Arithmetic Skills, since the latter two categories would also include individuals with problems in arithmetic procedures (e.g., borrowing). Thus, in the current study, we distinguish co-morbid DD from more general problems in arithmetic that frequently accompany ADHD.

\section{Neuroscience and Development of Numeracy Skills}

It has been shown that humans have at least two distinct means of representing and processing numerical information, which involve different neural networks [36,37]. One involves the left inferior frontal lobe and bilateral Angular gyri, which are associated with linguistic representation (e.g., numerical symbols), are activated during exact calculation, and serve a more supportive role in the management of successive arithmetic operations in working memory and in rote verbal memory. Another involves a specific part of the parietal brain region (horizontal intraparietal sulcus) that plays a central role in basic representation of numerical quantity, which is amodal and language-independent (e.g., 33,34). Notably, children with DD, who exhibit problems with basic processing of quantity, have been found to have anatomical and functional abnormalities in the intraparietal sulcus [38-40].

Moreover, it has been shown that simple subtraction involves manipulation of quantities and hence, activates a distinct neural network that includes the Intraparietal Sulcus (area that is known to be involved with mental basic processing of quantities, e.g., 19,41, 42).

The documentation of distinct neural substrates for different aspects of number processing suggests that perturbations in one or more networks may give rise to different profiles of arithmetic impairments. Moreover, if psychotropic medication differentially influences various brain circuits or regions, it may selectively improve performance on some but not all aspects of arithmetic performance.

\section{Rationale, Objectives and Hypotheses of Present Study}

The objective of this study was to investigate effects of stimulant medication on arithmetic performance in children with ADHD. We identified three groups of children with ADHD from an existing large data base: one group with DD (ADHD+DD), a group with more general and less severe difficulties in arithmetic (ADHD+MD), and a group with good arithmetic abilities (ADHD).

We predicted [1] that arithmetic difficulties in children with ADHD+MD might arise primarily from deficits associated with general cognitive deficits (e.g., in executive function and working memory), whereas ADHD+DD might also exhibit specific deficits in understanding quantities, a deficit known to be associated with DD. [2] Given the fact that MPH is known to enhance general but not specific cognitive functions, we predicted that MPH would enhance performance on arithmetic problems dependent upon working memory (borrowing, involving activation in the Prefrontal areas, e.g., 43), but would not influence performance on simple subtraction problems that involve specific understanding of quantity (involving activation in the Parietal lobe, e.g., 18).

\section{METHOD}

\section{Recruited Sample and Selected Participants}

We analyzed data from 18 children who were selected from a large database of children $(n=170)$, all of whom had a confirmed DSM-IV diagnosis of ADHD and had completed the same research protocol in an acute randomized placebocontrolled cross-over trial of MPH (as described below). All had been referred for the stimulant trial by the clinical neuropsychiatry team in a large metropolitan pediatric health sciences centre. All children had a full-scale intelligence quotient (IQ) of at least 82, and none had current evidence or history of neurological dysfunction, poor physical health, uncorrected sensory impairments or a history or psychosis. 
For the present study, children were also required to have at least average reading scores. A total of 16 children were excluded because they scored below the 19th percentile (standard score of 87 -low average) on the Reading Subtest of the Wide Range Achievement Test - Third Edition ("WRAT3": 44) and 3 additional were excluded because of low IQ.

All participants had received the same clinical diagnostic assessment, which used DSM-IV criteria, and received a confirmed diagnosis of ADHD [45]. Details of the clinical assessment procedure have been described previously (e.g., 46). Briefly, children were included in the study if they met DSM-IV criteria for ADHD based on information obtained using a semi-structured interview for parents ("PICS-IV": 47), with supportive evidence from parent and teachers versions of standardized behavior rating scales. Also, the Wechsler Intelligence Scale for Children - Third Edition ("WISC-III": 48) and the Wide Range Achievement Test ("WRAT3": 44) were administered during the initial assessment session. Diagnoses were based on a research algorithm and were also consistent with clinical consensus diagnosis. All children were free of medication for a minimum of 24 hrs before diagnostic assessment and participation in the medication trial.

\section{Classification of Arithmetic Subgroups}

We excluded 53 additional children who had completed the two lowest level math worksheets, because those sheets did not include any problems involving multi-digit subtraction as required for the present study (see description below under section titled: "Math task"), leaving a potential pool of 98 participants from the original 170 for inclusion in the present study. The 18 participants in the current study were selected from the subset of 98 , on the basis of their arithmetic and reading performance profiles on the WRAT3 and classified into one of three groups. (1) ADHD group; (2) Comorbid ADHD+Mathematical Difficulties (ADHD+MD) group; and (3) Co-morbid ADHD+Developmental Dyscalculia (ADHD+DD) group. The demographic and clinical features are summarized in Table $\mathbf{1 .}$

\section{Inclusion Criteria for the ADHD+DD Group}

Diagnosis of DD is generally determined psychometrically on the basis of low scores on individually administered standardized tests of arithmetic achievement, but there is no consensus as to the threshold score to be used in decision making. Guided by the work of Shalev and colleagues [49], we used a stringent criterion for DD by only including children whose scores were below the 8th percentile (a standard score of 79 - borderline) on the WRAT3 Arithmetic calculation subtest (which includes counting, reading number symbols, solving oral problems, and doing written computations). The WRAT arithmetic is very diverse in its mathematical requirements but involves mainly the use of quantity knowledge (e.g., deciding which number is larger, simple subtractions and divisions). Hence, we assumed that children with deficits in processing quantities will have very low score due to problems in most of the items. A total of 6 children out of the original sample of $170(3.5 \%)$ met these criteria for $\mathrm{ADHD}+\mathrm{DD}$.

\section{Inclusion Criteria for the ADHD+ Math Difficulties Group $\underline{(A D H D+M D)}$}

This group included children whose scores fell below the 23rd percentile but above the 8th percentile (i.e., standard score between 80 and 89) on the WRAT3 Arithmetic calculation subtest. A total of 9 children $(9.1 \%)$ of the sample of participants met these criteria for ADHD+MD and from these we selected six to yield the best match with children with the ADHD+DD group in terms of age, reading and IQ scores, gender, and subtype.

\section{Inclusion Criteria for ADHD Group}

This group included a total of 83 children whose scores on the WRAT3-Arithmetic subtest were above the 25th percentile (a standard score of 90 or more; at least in the average range for arithmetic computation). We selected 6 children best matched to those in the other two groups.

Table 1. Sample Characteristics

\begin{tabular}{|c|c|c|c|c|c|c|}
\hline & $\mathbf{M}$ & SD & $\mathbf{M}$ & SD & $\mathbf{M}$ & SD \\
\hline Age (years) & 8.94 & 1.27 & 9.79 & 1.16 & 10.45 & 1.145 \\
\hline Full Scale IQ & 105.5 & 10.82 & 109.66 & 8.8 & 98.16 & 16.09 \\
\hline WRAT3:Reading & 100.5 & 10 & 96.84 & 4.79 & 100.33 & 11.12 \\
\hline Male/Female & $5 / 1$ & & $4 / 2$ & & $6 / 0$ & \\
\hline \multicolumn{7}{|l|}{ ADHD subtype ${ }^{a}$} \\
\hline Inattentive & \multicolumn{2}{|c|}{4} & \multicolumn{2}{|c|}{4} & 5 & \\
\hline
\end{tabular}

${ }^{a}$ Number of participants meeting DSM-IV criteria for the specified ADHD subtype.

${ }^{\mathrm{b}}$ Significant difference between groups; $(\mathrm{F}(2,4)=9.964, \mathrm{p}<0.03$. 


\section{Math Task}

\section{Graded Worksheets}

Math computation worksheets appropriate for children in grades one to six had been developed specifically for use in our acute randomized placebo-controlled within-subject medication trials (e.g., 7), based on work by Douglas and colleagues [21]. We had developed a screening form for each grade level (for subtraction) and five equivalent worksheets for use in the acute medication trial (one baseline day, three drug trial days and one placebo day). Order and type of problems (i.e., large vs. small numbers and signs) were kept constant throughout the 5 equivalent forms. Each math worksheet level was approximated to school grade level work and contained subtraction questions (ranging from 30\% to 50\% of computational items), with levels five and six including multiplication items as well.

The math worksheets were constructed so that the level of difficulty was increased across grade level by expanding the number of digits in each question and/or increasing the procedural demands (i.e., frequent borrowing). Items were organized in a manner that required the child to shift arithmetic operations (e.g., subtraction) within each row of five or six items. Also, components of the problems were repeated within worksheet (e.g., 7-5 occurs as 17-5).

The primary dependent measure for the subtraction problems was accuracy, which was broadly defined as the number of correct items divided by the total number of prespecified items that were attempted in 10 minutes. Based on the classification system of Rasanen and Ahonen [50] we preselected problems on the worksheets that involved 2 types of subtraction skills (see Fig. 1). Specifically, we distinguished between items involving simple subtraction items (subtracting smaller from larger number) from those requiring the procedure of 'borrowing' (subtracting larger from smaller number).

\section{Borrowing: General Cognitive}

Borrowing errors in the following cases: 4-1 and 2-1 in the following problem:

\begin{tabular}{rr}
45 & 128 \\
-19 & -19 \\
\hline 34 & 119
\end{tabular}

\section{Simple subtraction: Basic Cognitive}

9-5 and 2-1 in the following problem:

13

Fig. (1). Example of items requiring general cognitive functions: borrowing (i.e., subtracting larger numbers from smaller numbers, and remembering that quantities have been borrowed). Example for items requiring basic cognitive functions: simple subtraction facts.
It should be noted that this math sheets are very much different from the arithmetic tests in WRAT 3 were only 2 subtractions that include both borrowing and simple subtraction are introduced to children no matter what their math level is (i.e., 46-29 and 401-74).

\section{Drug Protocol}

Children were tested individually in a simulated classroom laboratory during a five-day randomized double-blind placebo controlled trial of methylphenidate (MPH). Testing occurred over a period of five consecutive days, Monday through Friday, for approximately three hours per session. In each session, participants completed the math task and other cognitive measures (not reported here). After baseline measures were obtained on the first day ("practice day"), each child received placebo and each of three doses $(10 \mathrm{mg}, 15 \mathrm{mg}$ and $20 \mathrm{mg}$ ). All children weighed at least $25 \mathrm{~kg}$. For the specific group of participants in the current study, this translated to the following mean $\mathrm{mg} / \mathrm{kg}$ for each of theMPHdose levels: low 0.27(0.01), medium 0.42(0.09) and high 0.58(0.12). Fixed doses of MPH were used because there is no clear evidence that response to medication is dependent on body weight.

Master randomization tables were prepared by the research support pharmacist at the hospital using simple randomization with restrictions (high dose not to be given on the first possible drug day nor immediately following placebo; no directly ascending or descending dose order). From 24 possible orders, these restrictions left 11 possible orders. Therefore a balanced block 22 ( 2 of each of the 11 possibilities) design was used. Two separate tables were prepared, one for the younger children (6 to 8 years) and one for the older children ( 9 to 12 years). Doses were administered in a counter-balanced order so that approximately equal numbers of children received each of the possible drug condition orders. Thus, approximately equal number of children in the 3 groups received placebo on day 2, 3, 4 and 5, permitting a post-hoc group comparison on placebo (non-medicated state) to validate our group classification system. The examiner, psychiatrist, child, and child's family were not informed about the child's randomization order or daily medication status until trial completion. Placebo and active medication was prepared by the hospital pharmacist, powdered and packaged in an opaque gelatin capsule to prevent identification of contents by color, taste or volume. Each child's medication was placed in an individually named and dated (i.e., Day 2, Day 3, Day 4 or Day 5) envelope to ensure accurate administration.

It should be noted that, Swanson and Volkow [51] have carried out a series of functional neuroimaging studies, using PET and radiolabled methylphenidate in both humans and primates, to study the pharmacokinetic properties of intravenous and oral methylphenidate and levels of dopamine transporter (DAT) blockade. These studies demonstrated that. After oral doses of MPH, peak brain levels occur between 1 and 2 hours after dosing, which is about the same time as the peak pharmacokinetic and pharmacodynamic (behavioural) effects of clinical doses.

\section{Procedure}

The mathematics task was presented approximately 1.5 hours after ingesting the capsule containing MPH or placebo: 
that is, during the peak period of activation for MPH. Each child was tested individually and provided with a math computation worksheet and a pencil with an eraser on each day of the medication trial. Children were instructed to work independently on the worksheet for a total of 10 minutes. The examiner sat approximately three feet away form the participant at a separate table to encourage independent effort by the child and to allow for observations of the child's behavior and possible side-effect of medication (no child completed the worksheet in the allotted time period). At the end of the 10-minute period, the tester circled the last problem completed by the child.

\section{Statistical Analysis}

To determine the effects of MPH on arithmetic performance, we conducted multivariate analysis of variance for repeated measures for children's subtraction scores. Subtraction analyses were based on a $3 \times 2 \times 4$ factorial design, with Group (3 levels) as the between-subjects variable, and type of item (borrowing vs. simple subtraction) and dose (4 levels) as within subjects repeated measures.

To examine the utility of our initial group classification procedure, which was based simply on arithmetic achievement scores, we conducted a post-hoc group comparison of children's arithmetic performance in the placebo (nonmedication) condition, since the baseline assessment procedure did not provide adequate data on unassisted performance. Subtraction skills were assessed in a 3 (group)x 2 (item type) analysis of variance for repeated measures.

\section{RESULTS}

\section{MPH Effects Subtraction}

Two main effects (Group, Dose) were significant. Correct responses were higher in the ADHD group (mean correct responses were $0.99,0.934$ and 0.779 for the ADHD, $\mathrm{ADHD}+\mathrm{MD}$ and $\mathrm{ADHD}+\mathrm{DD}$ respectively) $[F(2,15)=232$. $09, p<0.0001$,; partial $\left.\eta^{2}=0.963\right]$. Correct responses varied as a function of MPH dose (mean correct responses were $0.858,0.90,0.912$ and 0.94 for the Placebo, Low, Medium and High levels of MPH respectively) $[F(3,45)=13.89$, $p<0.0001$; partial $\left.\eta^{2}=0.881\right]$. Of note for our hypotheses, the triple interaction between Group, Level of MPH and Type of item was also significant $[F(6,45)=3.42, p=0.0072$; partial $\eta^{2}=0.819$ ] and is presented in Fig. (2). Separate analyses conducted for each group separately, revealed a significant interaction between Type of item and Dose Level for the ADHD+MD and ADHD+DD groups, but not for the ADHD group $[F(3,15)=4.065, p=0.026 ; F(3,15)=5.9, p<0.0072$ respectively]. In both the $\mathrm{ADHD}+\mathrm{MD}$ and $\mathrm{ADHD}+\mathrm{DD}$ groups, MPH influenced performance, but only on the Borrowing items (i.e., general cognitive function) [ADHD+MD: $F(3,15)=8.23, \quad p<0.001 ; \quad$ ADHD+DD: $F(3,15)=10.4$, $p<0.001]$. Specifically, in each one of the ADHD+MD and ADHD+DD groups, the number of correct response was higher as the dose level increased [ADHD+MD group: low compared to placebo $F(1,15)=14.59, p<0.01$; medium compared to placebo $F(1,15)=37.63, p<0.0001$; High compare to placebo $F(1,15)=26.8, p<0.001$; ADHD+DD group: low compare to placebo not significant: medium compare to placebo $F(1,15)=17.49, p<0.001$; high compared to placebo $F(1,15)=39.90, p<0.0001]$ Medication had no effect on performance on simple subtraction items. Separate analyses were also conducted for each item separately . Dose of MPH significantly interact with Group only in the borrowing items $[F(6,45)=5.74, p=0.001]$ but not in the simple subtraction items. However, due to the theoretical importance we further analyzed these two interactions (i.e., Group X Dose in each item). Borrowing items: Compare to the ADHD group, the ADHD+MD group had lower correct results only in the placebo $[F(1,15)=26.58, p<0.001)$ and the ADHD+DD had lower results compare to the ADHD in all but the high dose [placebo: $F(1,15)=36.57, p<0.0001$; low: $F(1,15)=29.59$, $p<0.0001$; medium: $F(1,15)=25.29, p<0.0001]$. Simple subtraction items: No significant difference was found between the ADHD and the ADHD+MD groups in the simple subtraction items. However, the ADHD+DD had lower number of correct responses compare to the ADHD group in all the 4 doses [placebo: $F(1,15)=87.04, p<0.0001$; low: $F(1$, $15)=45.89, p<0.0001$; medium: $F(1,15)=197.63, p<0.0001$; high: $F(1,15)=64.60, p<0.0001]$.

\section{Basic cognitive function: Simple subtraction}

General cognitive function: Borrowing

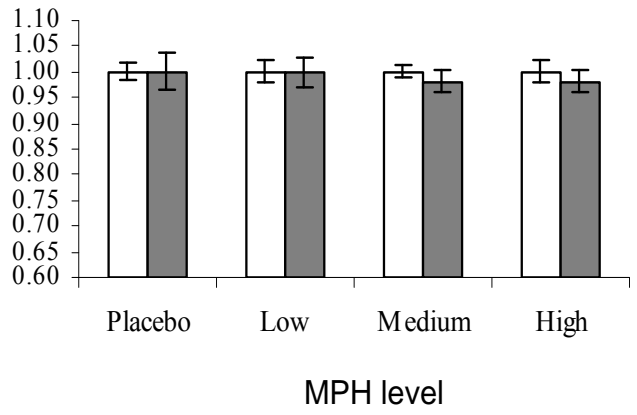

ADHD group

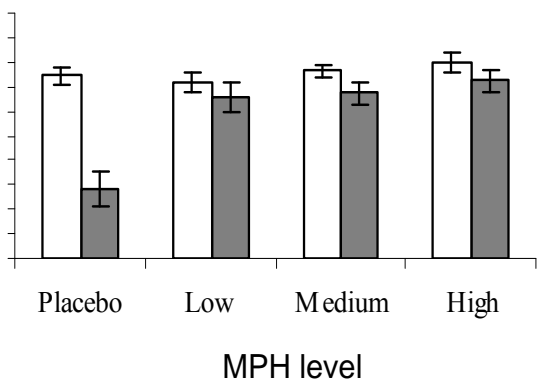

ADHD+MD group

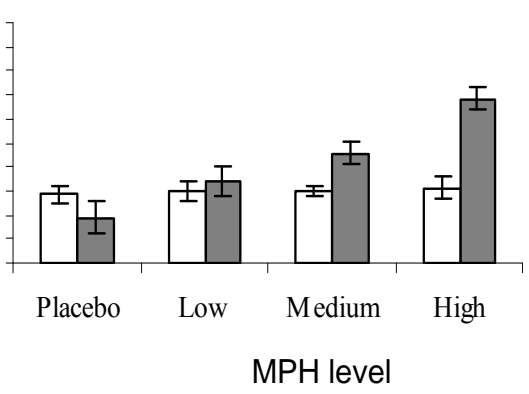

ADHD+DD group

Fig. (2). Subtraction: Squared interaction between Group X Type of item X MPH levels. 


\section{Group Differences in Unmedicated (Placebo) State}

As shown in Table 2, group comparisons on performance in an unmedicated (placebo) state revealed significant main effect for Group $[F(2,15)=38.23, p<0.0001]$ and post-hoc analysis revealed that, overall, the ADHD+DD performed worse than the ADHD+MD group $[F(1,15)=18.16, p<0.001]$. Also the two-way interaction between Group and Type of items was significant $[F(2,15)=13.9, p<0.001$ - for description of significance levels of this interaction see Table 2].

Table 2. Proportion of Items Correct for the Different Types of Arithmetic Problems for Each of the ADHD Subgroups, in Placebo Condition

\begin{tabular}{|c|c|c|c|}
\hline Problem Type & ADHD & ADHD+MD & ADHD+DD \\
\hline \hline $\begin{array}{c}\text { Basic arithmetic facts } \\
\text { Simple subtraction }\end{array}$ & $1.00^{\mathrm{a}}$ & $0.97^{\mathrm{a}}$ & $0.74^{\mathrm{c}}$ \\
\hline $\begin{array}{c}\text { Arithmetic Procedures } \\
\text { Borrowing (subtraction) }\end{array}$ & $1.00^{\mathrm{a}}$ & $0.74^{\mathrm{b}}$ & $0.69^{\mathrm{c}}$ \\
\hline
\end{tabular}

Groups with different superscripts differ significantly $(\mathrm{p}<.001)$.

\section{DISCUSSION}

This study yielded three novel findings, each of which were consistent with the hypothesised differential effects of stimulant medication on arithmetic computation and arithmetic subtypes in ADHD. First, our findings revealed that MPH enhanced performance on arithmetic procedures (i.e., borrowing) but had no effect on basic arithmetic facts. Second, MPH had differential efficacy for arithmetic performance in children with ADHD+MD versus ADHD+DD. Third, differences in the pattern of arithmetic performance in a nonmedicated state were observed between the three subgroups of children with ADHD.

MPH was found to have differential efficacy for arithmetic procedures 'borrowing') versus basic operations (simple subtraction involving number facts of 10). This pattern of effects suggests that previously reported beneficial effects of stimulants on arithmetic performance (e.g., 22) may be indirect and mediated by its influence on neural networks subserving general cognitive executive functions, such as working memory. The observed pattern of selective effects of MPH on component arithmetic abilities is consistent with its selective effects on some but not all aspects of cognitive performance [26,52].

The present findings also indicated that MPH has differential efficacy for children with ADHD+DD versus those with ADHD+MD. Specifically, MPH ameliorated all measured aspects of arithmetic calculation in children with ADHD+MD. By contrast, although MPH improved arithmetic procedures in children with ADHD+DD, it had no effects on their impaired performance on problems involving simple subtraction. One plausible explanation for the differential drug effect on this component skill in the two ADHD subgroups is that in the case of borrowing the children may still be using strategies that implicate working memory (43), but to do simple subtraction they use their implicit knowledge of quantity to simple subtractions (e.g., 18). Quantity processing would be intact in the ADHD+MD group but not in the ADHD+DD group. Thus, beneficial effects of MPH on working memory would improve performance in both groups but would not improve simple subtraction in the ADHD+DD group whose poor performance arises primarily from impairments in basic numeracy skills. The current results are supportive of the notion that DD population has deficits in basic numerical ability $[14,35]$. That is, the ADHD+DD group could not efficiently use quantity information and do simple subtraction due to deficits in core knowledge of quantities.

Conclusions based on the present findings are limited by several factors. First, we could only test small sample of participants and only a limited number of relevant arithmetic problems were available for analysis. However, it should be noted that despite this limitation, significant differences of large effect-size were found (values of partial $\eta^{2}$ ranged from 0.819 to 0.963 ). Second, because it was a child sample we do not know if the same results would hold for adolescents or adults. Third, the findings were based on an acute challenge with stimulant and may not reflect the outcome from a maintenance regimen of stimulant treatment. On the other hand, the Multimodal Treatment Study for Attention-Deficit Hyperactivity Disorder [24] showed that 14 months of carefully monitored treatment with stimulant medication had no effects on arithmetic achievement scores. Nonetheless, replication with larger clinical samples, more extensive arithmetic protocols, and longer-term treatment with stimulant medication, is required. This could direct a future development of a detailed cognitive description of DD, tests for the diagnosis of DD and remediation methods, which target children's core deficits.

To our knowledge, this is the first study (albeit smallscale) to demonstrate differential effects of MPH on arithmetic performance in children with ADHD plus general math problems versus those with co-morbid DD. Despite their limitations, these findings suggest that it is important from a clinical perspective to distinguish between ADHD+DD and ADHD+MD. Arithmetic difficulties in these two subgroups are attributable to different underlying cognitive problems that probably implicate dysfunction of different neural networks. The use of the severity of performance on standardized tests of arithmetic computation maybe useful tools for screening for DD. Also, these findings suggest that although stimulant treatment might ameliorate poor arithmetic performance as well as behavioral symptoms in the ADHD+MD children, those with ADHD+DD will need additional, intensive academic intervention. Notably, recent computerized training programs have been developed specifically for children with DD, and initial results are promising $[53,54]$.

\section{ACKNOWLEDGEMENTS}

This research was completed while Dr. Rubinsten was a post-doctoral fellow at Hospital for Sick Children (HSC), Toronto, Canada, and supported by the Rothschild Fellowship from Israel. It was undertaken, in part, thanks to funding from the Canadian Institutes of Health (CIHR: Grant \#MOP 64312), a CIHR post-doctoral fellowship (A-CB), and the Canada Research Chairs Program (RT). The authors thank 
Dr. Abel Ickowicz for his clinical support of the participants and the research.

\section{REFERENCES}

[1] Skounti M, Philalithis A, Galanakis E. Eur J Pediatr 2007; 166: 117-23.

[2] Spira EG, Fischel JE. J Child Psychol Psychiat 2005; 46: 755-773.

[3] Mayes SD, L. C.S. Learn Individ Diff 2006; 16: 145-157.

[4] Rivera-Batiz FL. J Hum Resour 1992; 27: 313-328.

[5] Biederman J, Mick E, Fried R, Aleardi M, Potter A, Herziq K. Psychiat Services 2005; 56: 1617-1620.

[6] Barry TD, Lyman RD. Klinger LG. A J School Psychol 2002; 40: 259-283.

[7] Benedetto-Nasho E, Tannock R. J Attention Disord 1999; 3: 121134.

[8] Zentall SS, Smith YN, Lee YB, Wieczorek C. J Learn Disabil 1994; 27: 510-519.

[9] Shalev RS. J Child Neurol 2004; 19: 765-771.

[10] Geary DC. J Learn Disabil 2005; 38: 305-307.

[11] Barkley RA. Psychol Bull 1997; 121: 65-94.

[12] Castellanos FX, Sonuga-Barke E.J.S, Milham MP, Tannock R. Trend Cognit Sci 2006; 10: 117-123.

[13] Passolunghi MC, Marzocchi GM, Fiorillo F. Devel Neuropsychol 2005; 28: 731-753.

[14] Butterworth B. J Child Psychol Psychiat 2005; 46: 3-18.

[15] Rubinsten O, Henik A. Neuropsychology 2005; 19: 641-648.

[16] Kobayashi T, Hirak K, Hasegawa T. Devel Sci 2005; 8: 409-419.

[17] Dehaene S, Molko N, Cohen L, Wilson AJ. Curr Opin Neurobiol 2004; 14: 218-224.

[18] Dehaene S. Cognitioin 1992; 44.

[19] Ischebeck A, Zamarian L, Siedentop C, Koppelstatter F, Benke T, Felber A, Delazer M. NeuroImage 2006; 30: 1365-1375.

[20] Zuvekas SH, Vitiello B, Norquist GS. Am J Psychiat 2006; 163: 579-585.

[21] Douglas VI, Barr RG, O'Neill ME, Britton BG. J Child Psychol Psychiat 1986; 27: 191-211.

[22] Silva R, Muniz R, Pestreich LK, Brams M, Childress A, Lopez FA. J Child Adolescent Psychiat 2005; 15: 637-654.

[23] Hechtman L, Abikoff H, Klein RG, Weiss G, Respitz C, Kouri J, Blum C, Greenfield B, Etcovitch J, Fleiss K. et al. J American Academy of Child and Adolescent Psychiatry 2004; 43: 812-819.

[24] MTA Cooperative Group. Pediatrics 2004; 113: 754-761.

[25] Grizenko N, Bhat M, Schwartz G, Ter-Stepanian M, Joober R. J Psychiat Neurosci 2006; 31: 46-51.

[26] Balthazor MJ, Wagner RK, Pelham WE. J Abnormal Child Psychol 1991; 19: 35-52.

[27] Muller U, Suckling J, Zelaya F, Honey G, Faessel H, Williams SCR, Routledge C, Brown J, Robbins TW, Bullmore ET. Psychopharmacology 2005; 180: 624-633.

[28] Bedard AC, Martinussen R, Ickowicz A, Tannock R. J Am Acad Child Adolescent Psychiat 2004; 43: 260-268.
[29] Volkow ND, Wang GJ, Fowler JS, Telang F, Maynard L, Logan J, Gatley SJ, Pappas N, Wong C, Vaska P. et al. Am J Psychiat 2004; 161: 1173-1180.

[30] Tannock R, Ickowicz A, Schachar R. J Am Acad Child Adolescent Psychiat 1995; 34: 886-896.

[31] Tannock R, Martinussen R, Frijters J. J Abnormal Child Psychol 2000; 28: 237-252.

[32] Arnsten AF. Neuropsychopharmacology 2006; 31: 2376-2383.

[33] Cohen Kadosh R, Cohen Kadosh K, Kaas A, Henik A, Goebel R. Neuron 2007; 53: 307-314.

[34] Piazza M, Pinel P, Le Bihan D, Dehaene S. Neuron 2007; 53: 293 305.

[35] Rubinsten O, Henik A. J Educat Psychol 2006; 98: 854-867.

[36] Dehaene S, Piazza M, Pinel P, Cohen L. J Cognitive Neurosci 2003; 20: 487-505.

[37] Eger E, Sterzer P, Russ MO, Giraud AL, Kleinschmidt A. Neuron 2003; 37: 719-725.

[38] Kucian K, Leoenneker T, Dietrich T, Dosch M, Martin E, von Aster M. Behav Brain Funct 2006; 5: 31.

[39] Molko N, Cachia A, Rivie're D, Mangin JF, Bruandet M, Le Bihan D, Cohen L, Dehaene S. Neuron 2003; 40: 847-858

[40] Cohen Kadosh R, Cohen Kadosh K, Schuhmann T, Kaas A, Sack AT. (in press) Curr Biol.

[41] Kong J, Wang C, Kwong K, Vangel M, Chua E, Golub R. Brain Res Cognitive Brain Res 2005; 22: 397-405.

[42] Delazer M, Benke T, Trieb T, Schocke M, Ischebeck A. Neuropsychlogia 2006; 44: 1909-1913.

[43] Geary, DG. Psychol Bull 1993; 114: 345-362.

[44] Wilkinson GS. The Wide Range Achievement Test - third addition (WRAT3) 1993. Psychological Corporation, San Antonio, TX.

[45] American Psychiatric Association. Diagnostic and statistical manual of mental disorders (DSM-IV). 1994; American Psychiatric Association, Washington, DC.

[46] Martinussen R, Tannock R. J Clin Exp Neuropsychol 2006; 28 : 1073-1094.

[47] Ickowicz A, Schachar RJ, Sugarman R, Chen SX, Millette C, Cook L. Canadian J Psychiat 2006; 51: 325-328.

[48] Wechsler D. Wechsler Intelligence Scale for Children 1991; Psychological Corporation, New York.

[49] Shalev RS, Manor O, Amir N, Gross-Tsur V. Devel Med Child Neurol 1993; 35: 593-601.

[50] Rasanen P, Ahonen T. Devel Neuropsychol 1995; 11: 275-295.

[51] Swanson J, Volkow N. In Stimulant drugs and ADHD 2001; (eds MV. Solanto, AF. Arnsten, \& F. X. Castellanos), pp. 259-282. New York: Oxford University Press.

[52] Pietrzak RH, Mollica CM, Maruff P, Snyder. PJ. Neuroscience and Behavioral Review 2006; 30: 1225-1245.

[53] Wilson AJ, Dehaene S, Pinel P, Revkin SK, Cohen L, Cohen D. Behav Brain Funct, 2.

[54] Wilson AJ, Revkin SK, Cohen D, Cohen L, Dehaene S. Behav Brain Funct 2006; 2.

(C) Rubinsten et al.; Licensee Bentham Open .

This is an open access article distributed under the terms of the Creative Commons Attribution License (http://creativecommons.org/licenses/by/2.5/), which permits unrestrictive use, distribution, and reproduction in any medium, provided the original work is properly cited. 